\title{
Initial Experience With a Radiology Imaging Network to Newborn and Intensive Care Units
}

\author{
Robert M. Witt, Mervyn D. Cohen, and C. Robert Appledorn
}

\begin{abstract}
A digital image network has been installed in the James Whitcomb Riley Hospital for Children on the Indiana University Medical Center to create a limited all digital imaging system. The system is composed of commercial components, Philips/AT\&T CommView system, (Philips Medical Systems, Shelton, CT; AT\&T Bell Laboratories, West Long Branch, $\mathrm{NJ}$ ) and connects an existing Philips Computed Radiology (PCR) system to two remote workstations that reside in the intensive care unit and the newborn nursery.

The purpose of the system is to display images obtained from the PCR system on the remote workstetions for direct viewing by referring cliniclans, and to reduce many of their visits to the radiology reading room three floors away. The design criteria includes the ability to centrally control all image management functions on the remote workstations to relieve the clinicians from any image management tasks except for recalling patient images. The principal components of the system are the Philips PCR system, the acquisition module (AM), and the PCR interface to the Data Management Module (DMM). Connected to the DMM are an Enhanced Graphics Display Workstation (EGDW), an optical disk drive, and a network gateway to an ethernet link. The ethernet network is the connection to the two Results Viewing Stations (RVS) and both RVSs are approximately $100 \mathrm{~m}$ from the gateway. The DMM acts as an image file server and an image archive device. The DMM manages the image data base and can load images to the EGDW and the two RVSs.

The system has met the initial design specifications and can successfully capture images from the PCR and direct them to the RVSs. Additional studies are beginning to determine the optimal image management procedures such as when to archive and purge images from the DMM.
\end{abstract}

Copyright 1991 by W.B. Saunders Company

KEY WORDS: PACS, PACS evaluation, image network.

$\mathbf{T}$ HE JAMES WHITCOMB Riley Hospital for Children is a tertiary care facility providing medical care to all children in Indiana and is part of Indiana University Medical Center. The facility has 262 beds with a large radiology facility staffed by three staff radiologists, two junior staff radiologists, and three residents. The radiology department is a new facility with 13 equipped rooms. The imaging modalities are three fluoroscopic rooms, computed radiography (CR), computed tomography, digital radiography, ultrasound, and nuclear medicine. Magnetic resonance imaging facility is adjacent to the Riley Hospital in a free standing building, but connected by an enclosed walkway. Approximately 70,000 imaging procedures are performed annually and of the diagnostic radiology procedures, approximately $17,000(28 \%)$ are portable examinations. Presently about $75 \%$ of the portable imaging procedures are processed through the PCR system (Table 1).

During the planning and construction of the new radiology facilities, implementation of a image network system connecting the digital modality of $\mathrm{CR}$ was added to the long-term facility plan. In pediatric radiology, the care of critically ill and premature infants often requires very rapid turnaround of images from the intensive care facilities to help monitor the progress of these patients. The most common image is a chest radiograph, which represents approximately $90 \%$ of the portable imaging procedures. The newborn nursery and the intensive care units (ICUs) are located approximately $100 \mathrm{~m}$ from radiology and are two and three floors away, respectively. The CR system provides the capability to obtain direct digital images of all portable chest images made in these intensive care facilities. The image network system provides the capabilities to return the images directly to these facilities for viewing by the attending staff. The image network could eliminate the need for attending staff to travel to radiology just to view more recent images. If necessary, consultations are completed by telephone.

\section{SYSTEM DESCRIPTION}

The principal components of our image management system are the Philips Computed Radi-

From the James Whitcomb Riley Hospital for Children, Indiana University Medical Center, Indianapolis, and The Department of Veterans Affairs, Richard L. Roudebush Medical Center, Indianapolis, IN.

Address reprint requests to Robert $M$. Wilt, $P h D$, Department of Veterans Affairs, Richard L. Roudebush Medical Cenier, 1481 W I0th St, Indianapolis, IN 46202.

Copyright 1991 by W.B. Saunders Company

0897-1889/91/0401-0002\$03.00\%0 
Table 1. Radiology Procedures Riley Hospital

\begin{tabular}{lrr}
\hline \multicolumn{3}{c}{ Annual Totals } \\
\hline Radiology procedures & 60,368 & $\{100 \%\}$ \\
Portable procedures & 17,066 & $\{28 \%\}$ \\
PCR/Graphic procedures & 12,800 & $\{21 \%\}$ \\
\hline
\end{tabular}

ology/Graphic (PCR) system and the Philips/ AT\&T CommView system (Philips Medical Systems, Shelton, CT; AT\&T Bell Laboratories, West Long Branch, NJ). The PCR system is a standard unit allowing the handling of $8 \times 10$ in, $10 \times 12$ in, and $14 \times 14$ in cassettes. The principal components of the PCR system are the Image Processor, the Image Reader, the Reception Terminal, the Examination Terminal, the PCR/CommView Interface Processor (PIP), a hard copy device, and two Data Management Systems (DMS), the DMS-10 and the DMS-20. The DMS-10 is the optical disk storage system and the DMS-20 is the Image Processing Console. The PCR/Graphic system allows operator interaction with the PCR and its locally stored images. The images at this point are still comprised of the raw data from the Image Processor and full image reprocessing is available to the operator. Operator reviewed and accepted images are sent to the CommView network via the PIP unit through the Acquisition module (AM). The images are received by the Data Management Module (DMM) for access by the CommView portion of the network and sent to the permanent archive (Fig 1).

The principal component of the CommView Network is the Data Management Module (DMM). This unit serves as a hub in a star network composed of fiber optic links to the other components. Connected to the DMM are an Enhanced Graphics Display Workstation (EGDW), the Acquisition Module (AM), and the CommView/Ethernet Gateway. The DMM local storage consists of both magnetic and optical disk drives. The DMM is the principal computer in the network and also has a cartridge tape drive for local backup and for loading system software. The CommView/ Ethernet Gateway provides the network link to the Results Viewing Stations (RVS). The RVSs are the image workstations located in the remote critical care facilities. The RVS is an AT\&T personal computer (Model 6312WGS) running DOS with a local ethernet communications board and a high-resolution display board to drive a $1 \mathrm{~K}$ by $1 \mathrm{~K}$ monochrome monitor. Data entry and control are via a keyboard or a mouse.

\section{METHODS}

The sources of data for reporting our initial experience with our PCR/CommView system are from the departmental log books of portable imaging procedures kept for the newborn nurseries and the ICUs. The data were sampled for

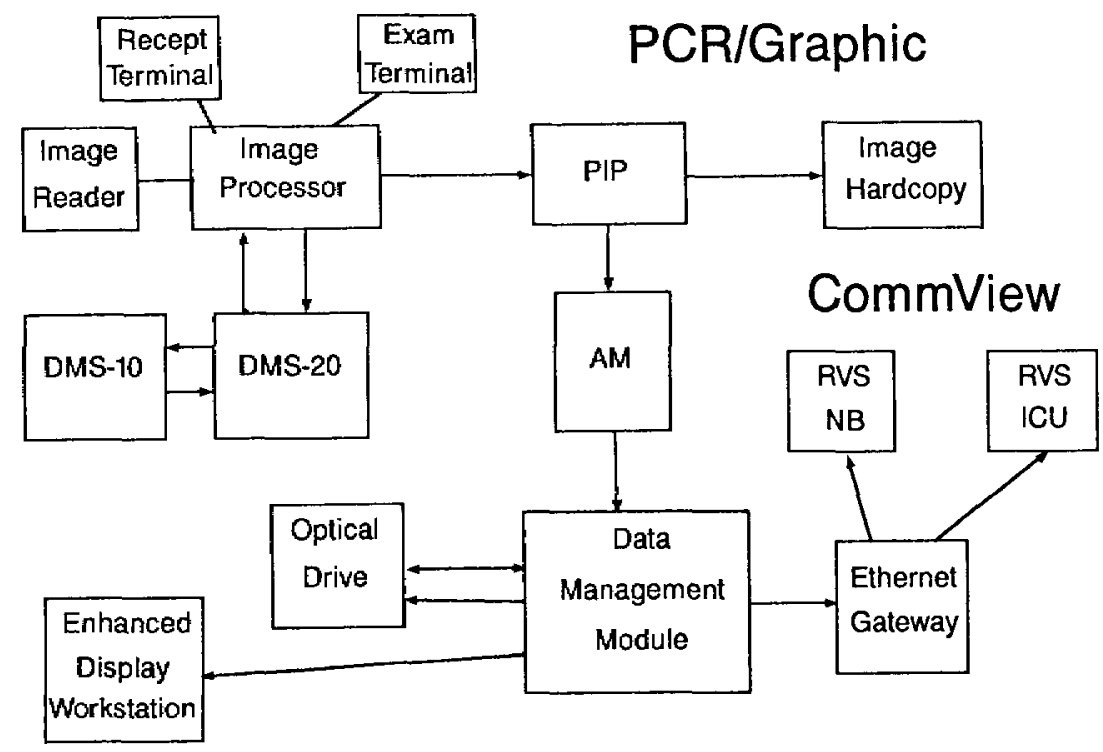

Fig 1. Diagram of the Image Network in the James Whitcomb Riley Hospital for Children. 
a 3-week period after the CommView network was operating for about 2 weeks with the most recent software update. The data accumulated for each day were the number of procedures or patients imaged, the size of the image receptor, and the time of day of the procedure. In the PCR/Graphic system, data is digitized to 12 bits or 4096 gray levels. The image data is compressed for transmission and storage with a no loss algorithm. The displays on the CommView display stations are 8 bits or 256 gray levels. The details of the data compression algorithm are not known, so the compressed image depth was assumed to be 8 bits. The amount of image data created each day and sent to the DMM was calculated. The average amount of image data created per day was calculated for both the newborn nursery and the ICUs for the sample period. The average amount of image data created per day was calculated for both facilities.

\section{RESULTS}

The results given in Table 2 summarize our initial observations. The number of patients/ procedures image per day in the newborn nursery ranged from 1 to 30 with an average of 17 patients per day. The number of patients/ procedure imaged per day in the ICUs ranged from 11 to 31 with an average of 23 patients per day. The number of megabytes of image data created per day in the newborn nursery ranged form 2.2 to 66.9 with an average of 37.4 . The number of megabytes of image data created per day in the ICUs ranged form 27.4 to 88.2 with an average of 63.4. The total amount of image data created per day for the two facilities was 100.8 Mbytes/d or approximately 0.1 Gbytes/d. From these estimates, 1 Gbyte of image data would be created each 10-day period so that at our current usage of the PCR/CommView system, we fill a 2.2 Gbyte optical disk on the DMM every 20 days.

Table 2. Results

\begin{tabular}{lccccc}
\hline Facility & \multicolumn{2}{c}{ Patients/d } & \multicolumn{2}{c}{ Image data/d (Mbyte) } \\
\hline \multirow{3}{*}{ Newborn } & Range & Average & Range & Average \\
ICU & 1 to 30 & 17 & 2.2 to 66.9 & 37.4 \\
Totals & 11 to 31 & 23 & 27.4 to 88.2 & 63.4 \\
\hline
\end{tabular}

\section{CONCLUSIONS AND DISCUSSION}

The PCR/CommView image management system has been in operation for approximately 3 months. In the last 2 months, the current release of the software allows the radiology technologist to direct images from the PCR/ Graphic system to a specific RVS. This last version of the software further satisfies our design specifications by allowing the system operator to purge images files stored on the RVS from the administrative terminal on the DMM.

Although the image management system as installed in the Riley Hospital only handles images generated from portable imaging procedures from just two facilities in our hospital, the ICUs and the Newborn Nursery, the image creation rate of 0.1 Gbytes/d overloaded the archive capabilities of the DMM. The design of the CommView system assumes that all images will be archived. A 2-Gbyte optical disk fills in approximately 20 days. When the image data storage elements fill completely, the system shuts down, including the PCR. For Riley, the temporal nature of these portable chest images does not require their long-term archive and storage. Typically only the current chest image and the next most recent image are required for review by the clinicians. The initial purpose of our image network was to provide remote access to the digital images created by the CR system for clinicians in those facilities not to provide for their long-term storage. The current release of the software does not allow the selection of individual images from the PCR for transfer to the optical disk archive on the DMM. However, a custom procedure automatically purges the image studies several times a day from the magnetic disk on the DMM maintaining sufficient storage for new images. The optical disk is currently not being used on the DMM. In the future, orthopaedic imaging procedures will be added to the studies done through the PCR, and long-term storage procedures may be implemented at that time.

The image management system installed in the Riley Hospital for Children performs all its tasks as designed, and can successfully capture images from the PCR and direct them to the proper RVS. It, however, has not been opera- 
tional long enough with sufficient reliability to monitor its impact on the clinical management of patients in the ICUs or to gauge its effect on utilization of physicians' and other care providers' time. As additional experience is gained with the system, it is our intention to monitor these activities and report the results at some future time.

\section{ACKNOWLEDGMENT}

The authors wish to thank Ms P. Copp and Ms S. Crago for their help in the preparation of this manuscript. 